\title{
Die Pulveraerosoldepositionsmethode - ein neues Verfahren zur Herstellung dichter Sensorschichten bei Raumtemperatur
}

\author{
Ralf Moos \\ Lehrstuhl für Funktionsmaterialien, Universität Bayreuth, Bayreuth, Deutschland \\ Kontakt: Funktionsmaterialien@uni-bayreuth.de
}

\section{Problemstellung}

Für Sensoren werden oft funktionskeramische Schichten benötigt. Da diese üblicherweise bei Sintertemperaturen über $1000{ }^{\circ} \mathrm{C}$ hergestellt werden, lassen sich kaum Keramiken mit niedrigschmelzenden Metallen, Gläsern oder Polymeren verbinden. Komplexere Funktionskeramiken lassen sich oft gar nicht erst herstellen, da sie sich zersetzen bevor sie verdichten.

\section{Das PAD-Verfahren}

Mit der Pulveraerosoldepositionsmethode (PAD) lassen sich dichte keramische Schichten ohne Hochtemperaturprozess direkt aus dem Ausgangspulver auf fast beliebige Substratmaterialien aufbringen. Dazu wird ein Pulveraerosol erzeugt und in ein Prozessgas geleitet. Das Aerosol wird durch eine Druckdifferenz vom Aerosolerzeuger in eine Vakuumkammer befördert, wo es durch eine Düse auf etwa Schallgeschwindigkeit beschleunigt und auf ein zu beschichtendes Substrat gelenkt wird (Abb. 1). Dabei können fest anhaftende Schichten von etwa $1 \mu \mathrm{m}$ bis ca. $100 \mu \mathrm{m}$ Dicke erzeugt werden. Die Funktionseigenschaften der erzeugten Schicht entsprechen in etwa denen des Ausgangsmaterials. Der Schichtbildungsmechanismus ist allerdings noch nicht völlig verstanden. Es wird angenommen, dass durch die große kinetische Energie die submikrometergroßen Partikel beim Aufprall auf das Substrat in nanometergroße Bruchstücke zerbrechen. Zunächst bildet sich eine Ankerschicht auf dem Substrat aus, danach kommt es zu einem kontinuierlichen Schichtaufbau bei gleichzeitiger Verdichtung („Hammering“) [1-3].

\section{Anwendungen}

Im Folgenden sollen zwei Anwendungsbeispiele aus dem Gebiet der Sensorik vorgestellt werden. Einerseits handelt es sich um NTC-Widerstände und anderseits um temperaturunabhängig resistive Sauerstoffsensoren.

\section{Mit dem PAD-Verfahren hergestellte NTC-Widerstände}

Auf Manganspinellen mit Zusätzen von Nickel und Kobalt basierende NTC-Widerstände sind bereits seit vielen Jahrzehnten auf dem Markt. Bis ca. $250{ }^{\circ} \mathrm{C}$ besitzen NTCThermistoren einige Vorteile. Zum Beispiel existiert ein breites Spektrum an verfügbaren Widerstandswerten $(1 \mathrm{k} \Omega$ bis $1 \mathrm{M} \Omega)$. Der betragsmäßig große Temperaturkoeffizient $\left(-20.000\right.$ ppmK $^{-1}$ bis -60.000 ppmK $^{-1}$; z. Vgl. Pt: +3.850 ppmK $^{-1}$ ) erlaubt Messungen von Temperaturdifferenzen $<0,1 \mathrm{mK}$ ohne großen schaltungstechnischen Aufwand.

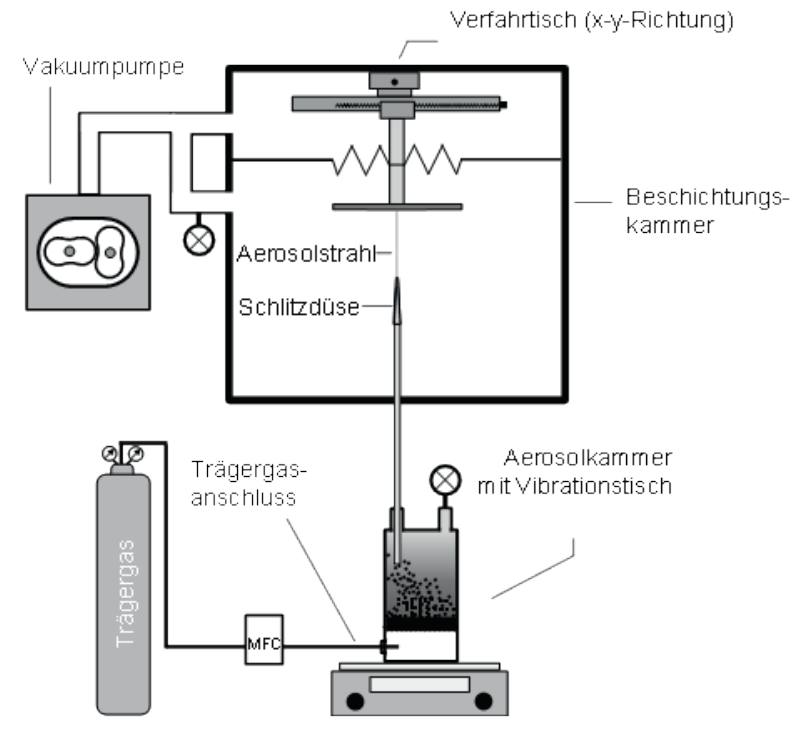

Abb. 1: Typischer Aufbau einer PAD-Anlage. Die aerosolerzeugende Einheit kann aus einer Rüttelflasche bestehen (einfacher Laboraufbau). Es können aber auch Pulveraerosolerzeuger verwendet werden, wie sie in der Partikeltechnik üblich sind.

In der Großserie stellt man NTC-Widerstände über klassische keramische Verfahren her. Dieser Gesamtprozess ist teuer und wenig flexibel. Die mechanische Bearbeitung setzt der Miniaturisierung Grenzen, weswegen mittels Schichtverfahren hergestellte Bauelemente von großem Interesse sind.

Hier bietet sich das oben beschriebene PAD-Verfahren an. Im ersten Schritt werden in klassischer Mischoxidtechnik $\mathrm{NiMn}_{2} \mathrm{O}_{4}$-Pulver mit kubischer Spinellstruktur hergestellt und durch Mahlen, Trocknen und Sieben so präpariert, dass sie sich danach gut im PAD-Prozess verarbeiten lassen. Mechanisch stabile, gut haftende, ca. $1 \mu \mathrm{m}$ dicke und homogene keramische Schichten mit kubischer Spinellstruktur lassen sich sehr gut abscheiden. Aufgrund der nanokristallinen Schichtstruktur und der vorhandenen Mikrodehnungen liegen die charakteristischen Kenngrößen NTC-Widerstände ( $\rho_{25}$ und $B$ ) zunächst etwas über denen von gesinterten Bulkkeramiken. Ein mildes nachträgliches Tempern der Schicht sorgt dafür, dass sich die Werte der $\mathrm{NiMn}_{2} \mathrm{O}_{4}$-Bulkkeramik wieder einstellen.

Hergestellt wurden Bauelemente der Chip-Baugröße 1206 (3,2 mm x 1,6 mm), wie in Abb. 2 gezeigt. 


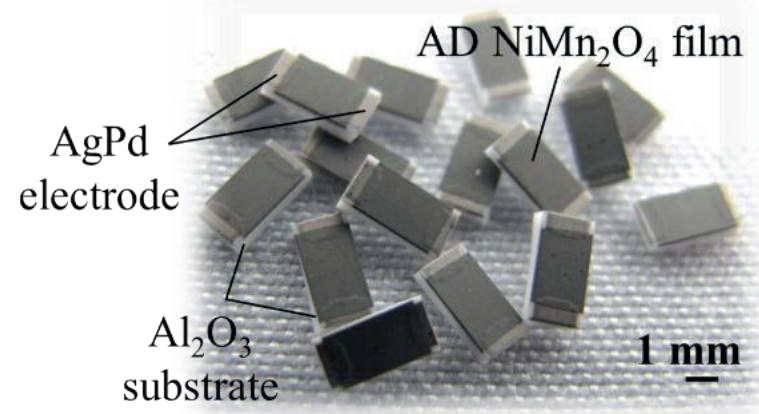

Abb. 2: NTC-Temperatursensoren bestehend aus einem Aluminiumoxidsubstrat, AgPd-Siebdruckelektroden und einer $\mathrm{NiMn}_{2} \mathrm{O}_{4}-\mathrm{NTC}$-Schicht, die mittels der PAD hergestellt wurde (aus [4]).

Die $B$-Werte streuten lediglich um max. $\pm 0,5 \%$, was den angestrebten Toleranzbereich von $\leq 1 \%$ deutlich übertraf. Beim $R_{25}$-Wert konnte eine Toleranz von $\pm 10 \%$ erreicht werden. Exakt passende Werte erhielt man danach nach einem Laserabgleich. Besonders vorteilhaft hat sich ein nachträgliches Tempern bei $400{ }^{\circ} \mathrm{C}$ herausgestellt, da sich dadurch auch die Alterungsstabilität der Schichten signifikant verbessert hat.

Weitere Details finden sich in den Arbeiten [5-8].

Mittels dem PAD-Verfahren hergestellte temperaturunabhängige resistive Sauerstoffsensoren.

Neben konventionellen konduktometrischen Gassensoren Sensoren aus $\mathrm{SnO}_{2}$ und anderen Metalloxiden zum Nachweis umweltschädlicher oder gefährlicher Substanzen, werden auch temperaturunabhängige resistive Sauerstoffsensoren diskutiert. Anwendung finden solche Sensoren in der Regelung und Überwachung von Verbrennungsprozessen bzw. deren Abgasnachbehandlungseinrichtungen. Mit Hilfe des PAD-Verfahrens wurde z.B. $\mathrm{SrTi}_{1-x} \mathrm{FexO}_{3}$ [9] oder $\mathrm{BaFe}_{1-\mathrm{x}} \mathrm{TaxO}_{3}$ (BFTx) eingesetzt [10]. Die simultane Co-Deposition von inerten und Sensorwerkstoffen bietet weitere vielversprechende Perspektiven zur Feinabstimmung der Sensoreigenschaften [11].

Eine etwas verbesserte Formulierung von BFT enthält 1 mol\% $\mathrm{Al}_{2} \mathrm{O}_{3}$ als Dotierstoff (BFATx). Unter den untersuchten BFATx-Proben wurden besonders gute Eigenschaften in Bezug auf die Temperaturunabhängigkeit des spezifischen Widerstandes für BFAT25 $\left(\mathrm{BaFe}_{0.74} \mathrm{Al}_{0.01} \mathrm{Ta}_{0.25} \mathrm{O}_{3}\right)$ gefunden. Abb. 3 zeigt ein typisches Messprotokoll bei $900{ }^{\circ} \mathrm{C}$. Dargestellt ist der Logarithmus der Leitfähigkeit (log $\sigma$ ) einer BFAT30-Schicht, die mit dem PAD-Verfahren hergestellt wurde. Das Material reagiert schnell und reproduzierbar auf den sich ändernden Sauerstoffpartialdruck $\left(p \mathrm{O}_{2}\right)$. Abbildung 4 zeigt die doppelt logarithmische Darstellung der Leitfähigkeit gegen den Sauerstoffpartialdruck des Sensors zwischen $600{ }^{\circ} \mathrm{C}$ und $900{ }^{\circ} \mathrm{C}$ im Sauerstoffpartialdruckbereich von 10 bis 1000 mbar.

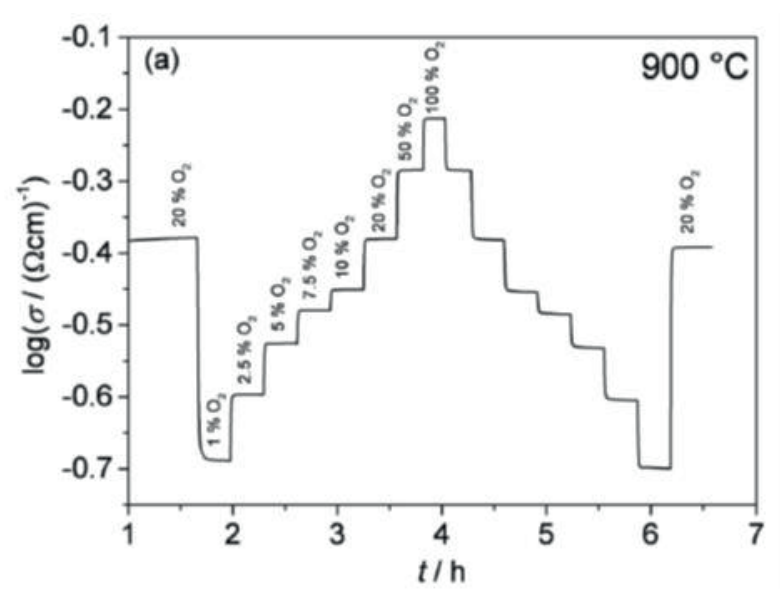

Abb. 3: Sensorsignal (Leitfähigkeit der Schicht $\sigma$ ) eines temperaturunabhängigen Sauerstoffsensors aus einem PAD-Film aus BFAT30. Aus [12].

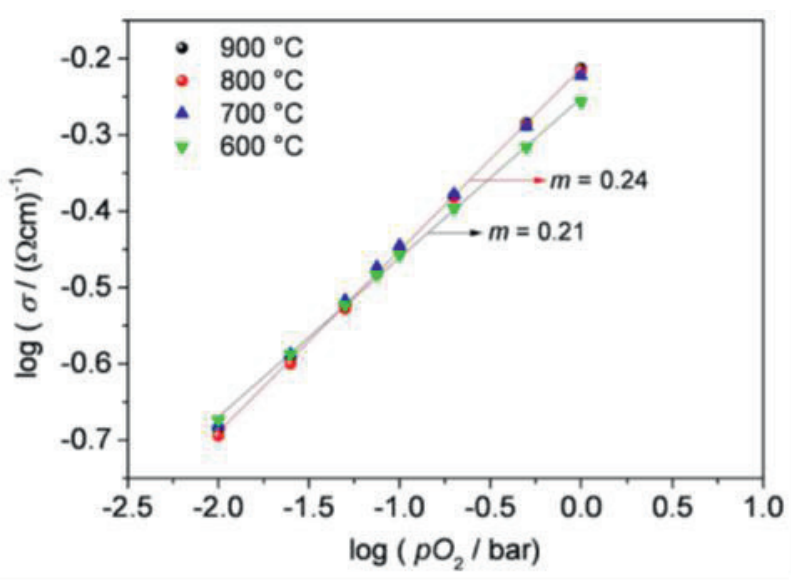

Abb. 4: Doppellogarithmischer Auftrag der Leitfähigkeit $\sigma$ gegen den Sauerstoffpartialdruck $p \mathrm{O}_{2}$ für einen PADbeschichteten BFAT30-Sensor. Man beachte, dass die Kurven von 700,800 und $900{ }^{\circ} \mathrm{C}$ sich kaum unterscheiden. Aus [12].

Weder die Empfindlichkeit gegenüber Sauerstoff (Steigung der Kennlinie) noch der Grundwiderstand ändern sich mit der Temperatur. Zwischen $700{ }^{\circ} \mathrm{C}$ und $900{ }^{\circ} \mathrm{C}$ hängt $\log \sigma$ linear von $\log \mathrm{pO}_{2}$ mit einer Steigung $m$ von $0,24 \mathrm{ab}$.

Sogar ein kombinierter resistiver und thermoelektrischer Sauerstoffsensor mit nahezu temperaturunabhängigen Eigenschaften sowohl der Leitfähigkeit als auch des Seebeck-Koeffizienten konnte hergestellt werden. Solch ein dualer thermoelektrisch-resistiver Sauerstoffsensor mit nahezu temperaturunabhängigen Eigenschaften beider Messgrößen wurde mit Hilfe von BFAT30 [13] auf einem Transducer abgeschieden, der eine Heizung, Äquipotentialschichten und Elektrodenstrukturen umfasst.

Auch fast alle anderen Metalloxidschichten können mit dem PAD-Verfahren bei Raumtemperatur aufgebracht 
werden. So zeigt klassisches Zinnoxid, wenn es auf Interdigitalelektroden abgeschieden wird, erwartungsgemäß eine ausgeprägte Empfindlichkeit auf Stickoxide bei etwa $300{ }^{\circ} \mathrm{C}$ und auf Wasserstoff bei etwa $450{ }^{\circ} \mathrm{C}$ [14].

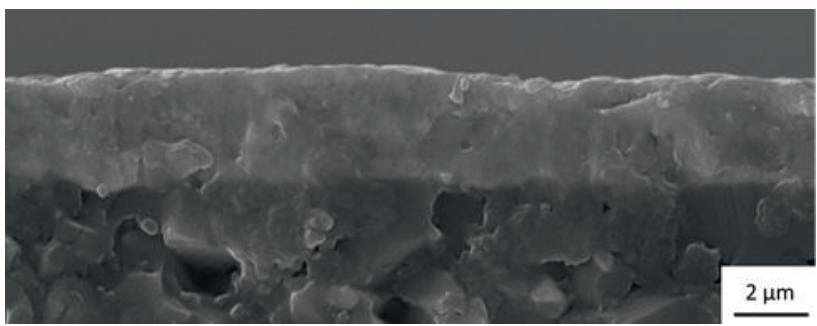

Abb. 5: Typische REM-Aufnahme (Bruch) einer keramischen Sensorschicht (hier: oxidionenleitendes BismutVanadat), die im PAD-Verfahren hergestellt wurde. Der PAD-Film (weniger dunkel) ist dicht und weist eine ausgezeichnete Verbindung mit dem AluminiumoxidSubstrat auf. Aus [1].

Die Pulveraerosoldepositionsmethode kann auch zur Herstellung elektrochemischer Gassensoren mit festen Elektrolyten verwendet werden. Ein Beispiel für einen typischen Stickoxidsensor nach der neuartigen PulsPolarisations-Methode, die in jüngster Zeit mit YSZ als Festelektrolyt viel Aufmerksamkeit erregt hat, ist in [15] gezeigt. Verwendet man einen Sauerstoffionenleiters auf Bismut-Vanadat-Basis mit höherer lonenleitfähigkeit kann der Sensor sogar bei noch niedrigeren Temperaturen betrieben werden. Der Aufbau ist in Abb. 4 gezeigt.

\section{Fazit}

Mit dem PAD-Verfahren ist es möglich, keramische Gassensorschichten ganz ohne Hochtemperaturprozess direkt aus einem keramischen Ausgangspulver auf fast jedem Substratmaterial herzustellen. Sogar Temperatursensoren können aufgebracht werden.

Zukünftige Arbeiten, die die Herstellung von Sensoren auf Polymer- oder Textilsubstraten zum Inhalt haben, sind bereits geplant.

\section{Danksagung}

Diese Übersichtsarbeit baut auf Beiträgen der gesamten PAD-Forschungsgruppe am Lehrstuhl für Funktionsmaterialien auf. Ganz herzlichen Dank für die tolle Arbeit!

\section{Literatur}

[1] D. Hanft, J. Exner, M. Schubert, T. Stöcker, P. Fuierer, R. Moos: An Overview of the Aerosol Deposition Method: Process Fundamentals and New Trends in Materials Applications, Journal of Ceramic Science and Technology, 6, 147-182 (2015), doi: 10.4416/JCST2015-00018

[2] J. Exner, T. Nazarenus, D. Hanft, J. Kita, R. Moos: What Happens during Thermal Post-Treatment of Powder Aerosol Deposited Functional Ceramic Films? Explanations Based on an Experiment-Enhanced Literature Survey,
Advanced Materials, 32, 1908104 (2020), doi: 10.1002/adma.201908104

[3] J. Exner, M. Schubert, D. Hanft, J. Kita, R. Moos: How to treat powders for the room temperature aerosol deposition method to avoid porous, low strength ceramic films, Journal of the European Ceramic Society, 39, 592-600 (2019), doi: 10.1016/j.jeurceramsoc.2018.08.008

[4] M. Schubert, D. Hanft, T. Nazarenus, J. Exner, M. Schubert, P. Nieke, P. Glosse, N. Leupold, J. Kita, R. Moos: Powder aerosol deposition method - novel applications in the field of sensing and energy technology, Functional Materials Letters, 12, 1930005 (2019), doi: 10.1142/S1793604719300056

[5] M. Schubert, C. Münch, S. Schuurman, V. Poulain, J. Kita, R. Moos, Thermal Treatment of Aerosol Deposited $\mathrm{NiMn}_{2} \mathrm{O}_{4}$ NTC Thermistors for Improved Aging Stability, Sensors, 18, 3982 (2018), doi: 10.3390/s18113982

[6] M. Schubert, C. Münch, S. Schuurman, V. Poulain, J. Kita, R. Moos, Characterization of Nickel Manganite NTC thermistor films prepared by Aerosol Deposition at room temperature, Journal of the European Ceramic Society, 38, 613-619 (2018), doi: 10.1016/j.jeurceramsoc.2017.09.005

[7] M. Schubert, J. Kita, C. Münch, R. Moos, Analysis of the characteristics of thick-film NTC thermistor devices manufactured by screen-printing and firing technique and by room temperature aerosol deposition method (ADM), Functional Materials Letters, 10, 1750073 (2017), doi: 10.1142/S1793604717500734

[8] M. Schubert, Aerosolbasierte Kaltabscheidung für die Herstellung von schichtbasierten NTCThermistorbauteilen. In: R. Moos, G. Fischerauer (Hrsg.), Bayreuther Beiträge zur Sensorik und Messtechnik, Bd. 29, Shaker-Verlag, Düren (2019), ISBN: 978-3-84407041-5

[9] K. Sahner, M. Kaspar, R. Moos, Assessment of the novel aerosol deposition method for room temperature preparation of metal oxide gas sensor films, Sensors and Actuators B: Chemical, 139, 394-399 (2009), doi: 10.1016/j.snb.2009.03.011

[10]M. Bektas, D. Schönauer-Kamin, G. Hagen, A. Mergner, C. Bojer, S. Lippert, W. Milius, J. Breu, R. Moos, BaFe 1 ${ }_{\mathrm{x}} \mathrm{Ta}_{\mathrm{x}} \mathrm{O}_{3-\delta}-\mathrm{A}$ material for temperature independent resistive oxygen sensors, Sensors and Actuators B: Chemical, 190, 208-213 (2014), doi: 10.1016/j.snb.2013.07.106

[11]J. Exner, M. Schubert, D. Hanft, T. Stöcker, P. Fuierer, R. Moos, Tuning of the electrical conductivity of $\mathrm{Sr}(\mathrm{Ti}, \mathrm{Fe}) \mathrm{O}_{3}$ oxygen sensing films by aerosol co-deposition with $\mathrm{Al}_{2} \mathrm{O}_{3}$, Sensors and Actuators B: Chemical, 230, 427-433 (2016), doi: 10.1016/j.snb.2016.02.033

[12]M. Bektas, D. Hanft, D. Schönauer-Kamin, T. Stöcker, G. Hagen, R. Moos, Aerosol-deposited $\mathrm{BaFe}_{0.7} \mathrm{Ta}_{0.3} \mathrm{O}_{3-\delta}$ for nitrogen monoxide and temperature-independent oxygen sensing, Journal of Sensors and Sensor Systems, 3, 223229 (2014), doi: 10.5194/jsss-3-223-2014

[13]M. Bektas, T. Stöcker, A. Mergner, G. Hagen, R. Moos, Combined resistive and thermoelectric oxygen sensor with almost temperature-independent characteristics, Journal of Sensors and Sensor Systems, 7, 289-297 (2018), doi: 10.5194/jsss-7-289-2018

[14]D. Hanft, M. Bektas, R. Moos, Powder pre-treatment for aerosol deposition of tin dioxide coatings for gas sensors, Materials, 11, 1342 (2018), doi: 10.3390/ma11081342

[15]J. Exner, G. Albrecht, D. Schönauer-Kamin, J. Kita, R. Moos, Pulsed Polarization-Based $\mathrm{NO}_{x}$ Sensors of YSZ Films Produced by the Aerosol Deposition Method and by Screen-Printing, Sensors, 17, 1715 (2017), doi: 10.3390/s17081715 